\title{
IRIS Recognition using Texture Features Extracted from Haarlet Pyramid
}

\author{
Dr.H.B.Kekre \\ Sr.Prof., \\ Computer Engg. Dept., \\ MPSTME, SVKM's, NMIMS, \\ Mumbai, India
}

\author{
Sudeep D. Thepade \\ Associate Prof., \\ Computer Engg. Dept., \\ MPSTME, SVKM's, NMIMS, \\ Mumbai, India
}

\author{
Juhi Jain , Naman Agrawal \\ MPSTME, SVKM's, NMIMS, \\ Mumbai, India
}

\begin{abstract}
Iris recognition has been a fast growing, challenging and interesting area in real-time applications. A large number of iris recognition algorithms have been developed for decades. The paper presents novel Haarlet Pyramid based iris recognition technique. Here iris recognition is done using the image feature set extracted from Haar Wavelets at various levels of decomposition. Analysis was performed of the proposed method, consisting of the False Acceptance Rate and the Genuine Acceptance Rate. The proposed technique is tested on an iris image database having 384 images. The results show that Haarlets level-5 outperforms other Haarlets, because the higher level Haarlets are giving very fine texture features while the lower level Haarlets are representing very coarse texture features which are less useful for discrimination of images in iris recognition.
\end{abstract}

\section{Keywords}

Iris recognition, Haarlet Pyramid, Haarlet Levels, False Acceptance Rate, Genuine Acceptance Rate.

\section{INTRODUCTION}

The term iris recognition refers to identifying, by computational algorithms, an unknown iris image. Iris recognition is a method of biometric authentication that uses pattern-recognition techniques based on high-resolution images of the irises of an individual's eyes. This operation can be done by means of comparisons between the unknown iris and iris images stored in the database.

In the area of human computer interaction, an ultimate goal is for machine to understand, communicate with and react to humans in natural ways. Although there are many other avenues to person identification - gait, clothing, hair, voice, and height are all useful indication of identity of the person, none are as compelling as iris recognition.

Iris recognition systems have a wide range of applications, especially when dealing with security applications, like computer and physical access control, real-time subject identification and authentication, and criminal screening and surveillance.

Retina scanning, iris recognition uses camera technology, with subtle infrared illumination reducing reflection from the convex cornea, to create images of the detail-rich, intricate structures of the iris. Converted into digital templates, these images provide mathematical representations of the iris that yield unambiguous positive identification of an individual.
Glasses or contact lenses do not interfere with the operation of iris recognition technology. Very few surgical procedures involve altering the iris, in which case re-enrolment in the database would be necessary. Blind people, as long as they have an iris present to scan, can likewise be identified with iris recognition technology.

Iris recognition technology offers the highest accuracy in identifying individuals as compared to any other method available. This is because no two irises are alike - not between identical twins, or even between the left and right eye of the same person. Irises are also stable; unlike other identifying characteristics that can change with age, the pattern of one's iris is fully formed by ten months of age and remains the same for the duration of their lifetime.

A key advantage of iris recognition is its stability, or template longevity, as, barring trauma, a single enrolment can last a lifetime. Because of its speed of comparison, iris recognition is the only biometric technology well-suited for one-to-many identification.

\section{HAAR TRANSFORM}

This sequence was proposed in 1909 by Alfréd Haar [13]. Haar used these functions to give an example of a countable orthonormal system for the space of square-integral functions on the real line. The study of wavelets, and even the term "wavelet", did not come until much later [14]. The Haar wavelet is also the simplest possible wavelet. The technical disadvantage of the Haar wavelet is that it is not continuous, and therefore not differentiable. This property can, however, be an advantage for the analysis of signals with sudden transitions, such as monitoring of tool failure in machines. The Haar wavelet's mother wavelet function $\psi(\mathrm{t})$ can be described as:

$$
\Psi(t)=\left\{\begin{array}{rr}
1 & , 0 \leq t<\frac{1}{2} \\
-1 & \frac{1}{2} \frac{1}{2} \leq t<1 \\
0 & { } \text { otherwise }
\end{array}\right.
$$

and its scaling function $\varphi(t)$ can be described as:

$$
\varphi(\mathrm{t})=\left\{\begin{array}{l}
1,0 \leq \mathrm{t}<1 \\
0, \text { otherwise }
\end{array}\right.
$$




\section{HAARLETS}

The procedure of generating Haarlets $[2,12]$ is shown in flowchart given in figure 1 and can be explained as given in following steps.

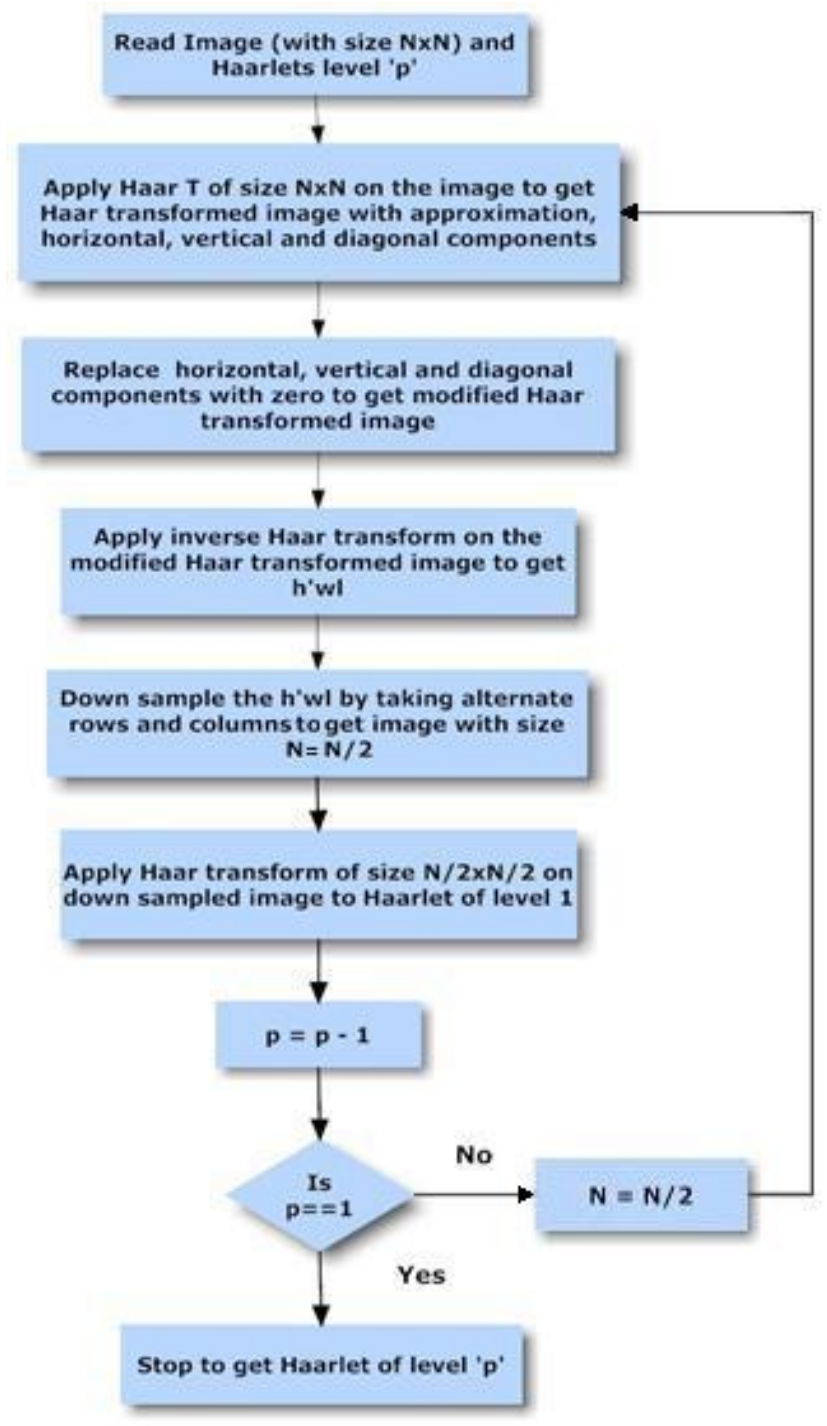

Fig. 1 Flowchart for generating Haarlets of level ' $p$ '

Let $\mathrm{I}_{\mathrm{NXN}}$ be the image with size $\mathrm{NxN}$ of which Haarlets are to be obtained and $\mathrm{H}_{\mathrm{NxN}}$ be the Haar transform matrix of size $\mathrm{NxN}$.

a) Apply Haar transform of size $\mathrm{NxN}$ on the image of size $\mathrm{NxN}$ to get Haar transformed image with approximation (hIA), horizontal (hIH), vertical (hIV) and diagonal (hID) components.

$$
\begin{aligned}
\mathrm{hI}_{\mathrm{NxN}} & =[\mathrm{hIA}, \mathrm{hIH}, \mathrm{hIV}, \mathrm{hID}] \\
& =\left[\mathrm{H}_{\mathrm{NxN}}\right]\left[\mathrm{I}_{\mathrm{NxN}}\right]\left[\mathrm{H}^{\prime}{ }_{\mathrm{NxN}}\right]
\end{aligned}
$$

b) Replace horizontal (hIH), vertical (hIV) and diagonal (hID) components with zero to get modified Haar transformed image 'hwI'.

$$
\mathrm{hwI}_{\mathrm{NxN}}=[\mathrm{hIA}, \text { Zero, Zero, Zero }]
$$

c) Apply inverse Haar transform on the modified Haar transformed image to get h'wI. $h^{\prime} \mathrm{wI}_{\mathrm{NxN}}=\left[\mathrm{H}^{\prime}{ }_{\mathrm{NxN}}\right]\left[\mathrm{hwI}_{\mathrm{NxN}}\right]\left[\mathrm{H}_{\mathrm{NxN}}\right]$

d) Down-sample the result of step'c' (h'wI) by taking alternate rows and columns to get image with size $\mathrm{N} / 2 \mathrm{xN} / 2$. $\mathrm{dhI}_{\mathrm{N} / 2 \times \mathrm{N} / 2}=$ down sample( h'wI $\left.\mathrm{NxN}\right)$

e) Apply Haar transform of size $\mathrm{N} / 2 \mathrm{xN} / 2$ on down-sampled image $\left(\mathrm{dwI}_{\mathrm{N} / 2 \times \mathrm{N} / 2}\right)$ to get the Haarlet of level1.

Haarlet Level I $=\left[\mathrm{H}_{\mathrm{N} / 2 \times \mathrm{N} / 2}\right]\left[\mathrm{dwI}_{\mathrm{N} / 2 \times \mathrm{N} / 2}\right]\left[\mathrm{H}^{\prime}{ }_{\mathrm{N} / 2 \times \mathrm{N} / 2}\right]$

f) Repeat steps b to e 'P-1' times on the level 1Haarlet to get Haarlet of level 'P'.

\section{HAARLETS PYRAMID}

The Haarlets of a particular image for different levels, when considered together gives Haarlet Pyramid [2]. Here for generating first level of Haarlet pyramid Haar transform is applied on image to get approximation, horizontal, vertical and diagonal components. The approximate components of first level Haarlet are considered to be transformed with Haar $\mathrm{T}$ to get second level Haarlet. The Haarlet pyramid of sample images are shown in figure 2 given below. Where the iris image is decomposed into three levels of Haarlet pyramid as Haarlet level-1, Haarlet level-2 and Haarlet level-3.

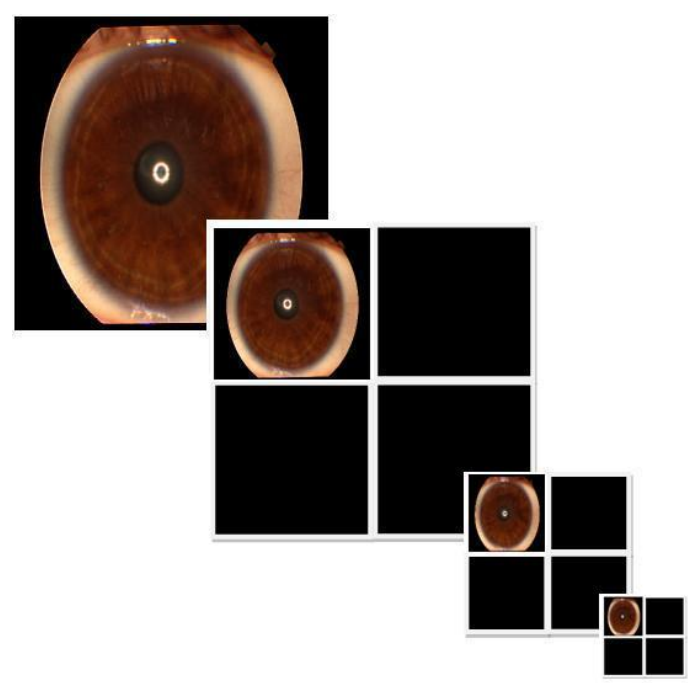

Fig. 2 Different Levels of Haarlet Pyramid

\section{EUCLIDEAN DISTANCE [3-9]}

We have used Euclidean distance [3-9] on the feature set as the similarity measure. The Direct Euclidian Distance between training image $\mathrm{P}$ and test image $\mathrm{Q}$ can be given as equation (6), where Vpi and Vqi be the feature vectors of training image $\mathrm{P}$ and test image $Q$ respectively of size ' $n$ '.

$$
E D=\sqrt{\sum_{i=1}^{n}(V p i-V q i)^{2}}
$$




\section{PROPOSED TECHNIQUES}

\subsection{Iris Image Feature Extraction}

Here the approximate components of Haarlet level-1, Haarlet level-2,....,Haarlet level-7 are obtained for every image in the database and Haar transforms of respective sizes are applied on them, the results are stored as feature vectors for respective image. At level-1 Haarlet the feature vector size is $\mathrm{N} / 2 \mathrm{xN} / 2$. At level-2 Haarlet the feature vector size is $\mathrm{N} / 4 \mathrm{xN} / 4$ and so on. Thus the feature vectors for upto level-7 Haarlets are extracted and the feature vector database is generated.

\subsection{Iris Recognition}

Here the feature set of Haarlet level-p is extracted as a feature set for query image using proposed technique of Haarlet generation. Then these are matched with Haarlet level-p feature vector database using Euclidian distance as similarity measure. As compared to applying complete Haar $\mathrm{T}$ on the image, this proposed method takes fewer computations to extract the feature set and gives better precision and recall values.

For image of size $\mathrm{NxN}$ complete Haar needs $2 \mathrm{~N}^{2} \log _{2}(\mathrm{~N})$ additions and for Haarlet of level-p the number of additions needed are $2\left(\mathrm{~N} / 2^{\mathrm{p}}\right)^{2} \log \left(\mathrm{N} / 2^{\mathrm{p}}\right)$ as the size of feature vector would be $\left(\mathrm{N} / 2^{\mathrm{p}}\right) \mathrm{x}\left(\mathrm{N} / 2^{\mathrm{p}}\right)$. This gives tremendous reduction in query execution time using higher Haarlet level.

\section{IMPLEMENTATION}

\subsection{Platform}

The experiments were performed on Matlab R2009b, Intel Core 2 Duo T8100 (2.1 Ghz).

\subsection{Database}

The techniques are tested on Iris Database created at Palacky University[18]. This database has 6x64 (i.e. 3x64 left and 3x64 right) iris images (each with 512 pixels by 512 pixels), corresponding to 64 persons, including both males and females. The irises were scanned by TOPCON TRC50IA optical device connected with SONY DXC- 950P 3CCD camera. The images were taken in a single session. The six images taken per person for three people are shown in Figure 3.

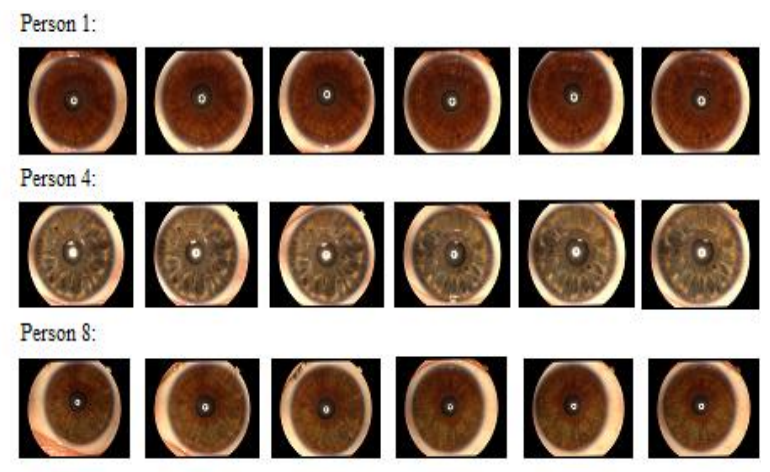

Fig. 3 Sample Images from Iris Database

\section{RESULTS \& DISCUSSIONS}

The false acceptance rate (FAR) [11] is the measure of the likelihood that the biometric security system will incorrectly accept an access attempt by an unauthorized user. A system's
FAR typically is stated as the ratio of the number of false acceptances divided by the number of identification attempts.

The genuine acceptance rate (GAR) [10] is the measure of the likelihood that the biometric security system will correctly accept an access attempt by an authorized user. A system's GAR typically is stated as the ratio of the number of correct acceptance divided by the number of identification attempts.

The FAR and GAR values were calculated for the comparison of all the images in the database. Average of the FAR and GAR values of the all the images in each level were taken together, forming the average FAR and GAR for the particular level.

During performance testing a test image was considered and five more images of the same person were compared and displayed, so percentage correct detection is the percentage of relevant images it returned and percentage incorrect detection is the amount irrelevant images it returned.

In all 384 queries were tested on our database for analysing the performance of proposed iris identification techniques. The table (TABLE 1) gives the percentage of FAR and GAR for iris identification using variations in Haarlet based techniques. Here it is observed that Haarlet-level-5 gives the best performance. It outperforms complete Haar transform and because of reduced feature vector size gives faster recognition.

TABLE 1: FAR/GAR for various Haarlet levels

\begin{tabular}{|r|r|r|}
\hline & GAR(in \%) & FAR(in \%) \\
\hline COMPLETE & 61.45833 & 38.54167 \\
\hline LEVEL 1 & 61.45833 & 38.54167 \\
\hline LEVEL 2 & 60.76389 & 39.23611 \\
\hline LEVEL 3 & 61.80556 & 38.19444 \\
\hline LEVEL 5 & 64.58333 & 35.41667 \\
\hline LEVEL 6 & 63.19444 & 36.80556 \\
\hline LEVEL 7 & 60.76389 & 39.23611 \\
\hline
\end{tabular}

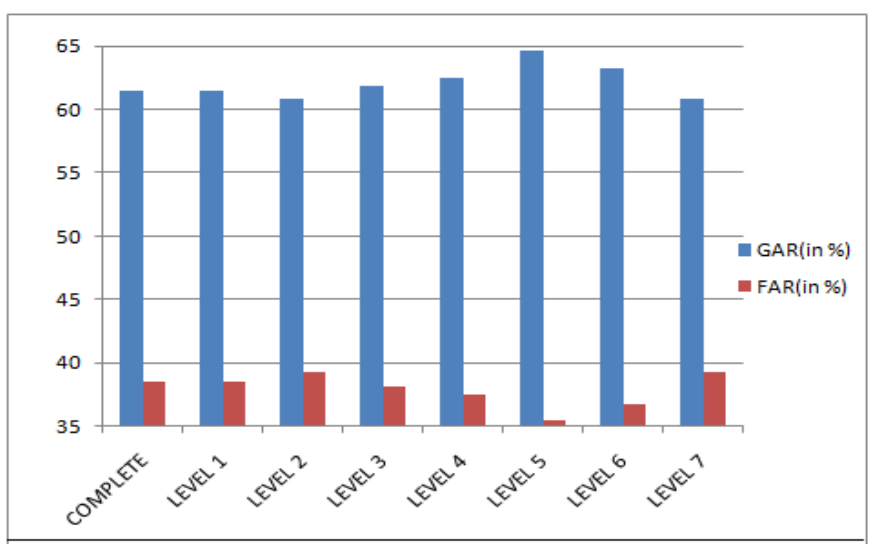

Fig.4 FAR/GAR Using Haarlets on Our database 


\section{CONCLUSION}

Recognition accuracy, robust method and computational costs are topics that must be taken into account when analyzing an iris recognition method. The FAR/GAR values show that Haarlets are outperforming Haar based image retrieval, proving that Haarlets has better discrimination capability. Also Haarlets of level-5 is giving better performance than other Haarlets because higher level Haarlets are giving very coarse texture features while lower level Haarlets are giving too fine texture features losing discrimination capability, which are not really useful in iris recognition and proper texture properties are carved by Haarlets level-5 at greatly reduced query execution time. The proposed method (Haarletslevel-5) is also suitable for real time applications.

\section{REFERENCES}

[1] Developed by Dr. Libor Spacek. Available Online at: http://cswww.essex.ac.uk/mv/otherprojects.html. [last referred on 10 Nov 2010]

[2] H.B.Kekre, Sudeep D. Thepade, "Image Retrieval using Color-Texture Features Extracted from Haarlet Pyramid", ICGST International Journal on Graphics, Vision and Image Processing (GVIP), Volume 10, Issue I, Feb.2010, pp.9-18, Available online www.icgst.com/gvip/Volume10/Issue1/P1150938876.html

[3] H.B.Kekre, Sudeep D. Thepade, "Creating the Color Panoramic View using Medley of Grayscale and Color Partial Images ", WASET International Journal of Electrical, Computer and System Engineering (IJECSE), Volume 2, No. 3, Summer 2008. Available online at www.waset.org/ijecse/v2/v2-3-26.pdf.

[4] Stian Edvardsen, "Classification of Images using color, CBIR Distance Measures and Genetic Programming", Ph.D. Thesis, Master of science in Informatics, Norwegian university of science and Technology, Department of computer and Information science, June 2006.

[5] H.B.Kekre, Tanuja Sarode, Sudeep D. Thepade, "DCT Applied to Row Mean and Column Vectors in Fingerprint Identification", In Proceedings of International Conference on Computer Networks and Security (ICCNS), 27-28 Sept. 2008, VIT, Pune.

[6] Zhibin Pan, Kotani K., Ohmi T., "Enhanced fast encoding method for vector quantization by finding an optimallyordered Walsh transform kernel", ICIP 2005, IEEE International Conference, Volume 1, pp I - 573-6, Sept. 2005.

[7] H.B.Kekre, Sudeep D. Thepade, "Improving 'Color to Gray and Back' using Kekre's LUV Color Space”, IEEE International Advanced Computing Conference 2009 (IACC'09), Thapar University, Patiala, INDIA, 6-7 March 2009. Is uploaded and available online at IEEE Xplore.

[8] H.B.Kekre, Sudeep D. Thepade, "Image Blending in Vista Creation using Kekre's LUV Color Space", SPIT-IEEE Colloquium and International Conference, Sardar Patel Institute of Technology, Andheri, Mumbai, 04-05 Feb 2008.
[9] H.B.Kekre, Sudeep D. Thepade, "Color Traits Transfer to Grayscale Images", In Proc.of IEEE First International Conference on Emerging Trends in Engg. \& Technology, (ICETET-08), G.H.Raisoni COE, Nagpur, INDIA. Uploaded on online IEEE Xplore.

[10] http://www.webopedia.com/TERM/F/false_rejection.html [last referred on 10 Nov 2010]

[11] http://www.webopedia.com/TERM/F/genuine_acceptance.ht $\mathrm{ml}$ [last referred on 10 Nov 2010]

[12] K.-C. Liang and C. C. Kuo, "WaveGuide: A Joint WaveletBased Image Representation and Description System," IEEE Trans. on ImageProcessing, vol. 8, no. 11, pp.1619-1629, 1999

[13] Haar, Alfred, "Zur Theorie der orthogonalen Funktionensysteme". (German), Mathematische Annalen, volume 69, No. 3, 1910, pp. 331-371.

[14] Charles K. Chui, "An Introduction to Wavelets”, Academic Press, 1992, San Diego, ISBN 0585470901.

[15] www.wisegeek.com/what-is-iris-recognition-technology.htm

[16] A. Basit, M. Y. Javed, M. A. Anjum, "Efficient Iris Recognition Method for Human Identification." Uploaded on online IEEE Xplore.

[17] Christel-loïc TISSE, Lionel MARTIN, Lionel TORRES, Michel ROBERT, "Person identification technique using human iris recognition." Uploaded on online IEEE Xplore.

[18] "http://www.advancedsourcecode.com/irisdatabase.asp" for Palacky University iris database.

\section{AUTHOR BIOGRAPHIES}

Dr. H. B. Kekre has received B.E. (Hons.) in Telecomm. Engg. from Jabalpur University in 1958, M.Tech (Industrial Electronics) from IIT Bombay in 1960, M.S.Engg. (Electrical Engg.) from University of Ottawa in 1965 and Ph.D. (System Identification) from IIT Bombay in 1970. He has worked Over 35 years as Faculty of Electrical Engineering and then HOD Computer Science and Engg. at IIT Bombay. For last 13 years worked as a Professor in Department of Computer Engg. at Thadomal Shahani Engineering College, Mumbai. He is currently Senior Professor working with Mukesh Patel School of Technology Management and Engineering, SVKM's NMIMS University, Vile Parle(w), Mumbai, INDIA. He ha guided 17 Ph.D.s, 150 M.E./M.Tech Projects and several B.E./B.Tech Projects. His areas of interest are Digital Signal processing and Image Processing. He has more than 300 papers in National / International Conferences / Journals to his credit. Recently six students working under his guidance have received best paper awards. Currently he is guiding seven Ph.D. students.

Sudeep D. Thepade has Received B.E.(Computer) degree from North Maharashtra University with Distinction in 2003. M.E. in Computer Engineering from University of Mumbai in 2008 with Distinction, currently Perusing Ph.D. from SVKM's NMIMS University, Mumbai. He has about 08 years of experience in teaching and industry. He was Lecturer in Dept. of Information 
Technology at Thadomala Shahani Engineering College, Bandra(w), Mumbai for nearly 04 years. Currently working as Assistant Professor in Computer Engineering at Mukesh Patel School of Technology Management and Engineering, SVKM's NMIMS University, Vile Parle(w), Mumbai, INDIA. He is member of International Association of Engineers (IAENG) and International Association of Computer Science and Information Technology (IACSIT), Singapore. His areas of interest are Image Processing and Computer Networks. He more than 90 papers in National/International Conferences/Journals to his credit with a Best Paper Award at International Conference SSPCCIN-2008 and Second Best Paper Award at ThinkQuest-2009 National Level paper presentation competition for faculty.
Juhi Jain is currently pursuing a Bachelors (B.Tech.) degree in Information Technology from MPSTME, SVKM's NMIMS University, Mumbai. Her areas of interest lie in the fields of Computer Networking, Image Processing and Biometrics.

Naman Agrawal is currently pursuing a Bachelors (B.Tech.) degree in Information Technology from MPSTME, SVKM's NMIMS University, Mumbai. His areas of interest lie in the fields of Database Management, Image Processing and Biometrics. 